\title{
Rehabilitation post-distraction osteogenesis for brachymetacarpia: a case report
}

\author{
Emily S. Ho ${ }^{1,2,5}$. Catharine S. Bradley ${ }^{3,5}$. Gregory H. Borschel ${ }^{1,4}$. Simon P. Kelley ${ }^{3,4}$
}

Received: 18 September 2017 / Accepted: 4 May 2018

(c) The Author(s) 2018

\begin{abstract}
Distraction osteogenesis for brachymetacarpia has been described in several small case series and single case reports, but the rehabilitation required to optimize outcomes has not been reported. We present a case report describing the hand rehabilitation program of a 13-year-old girl with congenital brachymetacarpia who underwent distraction osteogenesis of the third metacarpal. Intense weekly hand therapy including desensitization, scar massage, range of motion exercises and splinting was essential up to 28 weeks postoperatively to address the progressive changes in the anatomical structures. At final follow-up, she had full active range of motion, no functional deficits in grasp or in-hand manipulation skills and resumed her participation in competitive baton twirling. Patient and family satisfaction with outcome was high. However, better education regarding the progressive symptoms with distraction and daily challenges of wearing an external fixator would have improved the overall experience. With a strong family commitment to rehabilitation and thorough patient education, distraction osteogenesis for brachymetacarpia has the potential to improve functional and aesthetic outcome in the hand. Level of evidence $\mathrm{V}$.
\end{abstract}

Keywords Hand deformities $\cdot$ Congenital $\cdot$ Distraction osteogenesis $\cdot$ Adolescent $\cdot$ Case report $\cdot$ Rehabilitation

\section{Introduction}

Brachymetacarpia is a congenital condition characterized by undergrowth of the metacarpal bone in the hand [1]. This may be caused by idiopathic early closure of the epiphyseal plate [2]. Typically, when one digit is affected, the patient

Gregory H. Borschel and Simon P. Kelley have contributed equally to this work as senior authors.

Emily S. Ho

emily.ho@sickkids.ca

1 Division of Plastic and Reconstructive Surgery, The Hospital for Sick Children, 555 University Avenue, Rm 5433, Toronto, ON M5G 1X8, Canada

2 Rehabilitation Sciences Institute, University of Toronto, Toronto, Canada

3 Division of Orthopedic Surgery, The Hospital for Sick Children, Toronto, Canada

4 Department of Surgery, University of Toronto, Toronto, Canada

5 Department of Rehabilitation Services, The Hospital for Sick Children, Toronto, Canada may complain that the "knuckle is missing" due to severe recession of the affected metacarpal [3]. However, there are typically no significant functional issues although this recessed position may restrict metacarpal-phalangeal joint (MPJ) flexion [3]. Patients who pursue treatment for brachymetacarpia are likely seeking improvement in the cosmesis of their hand [2,3].

Distraction osteogenesis for brachymetacarpia has been described in several small case series and single case reports [2, 4-10]. The focuses of these cases are surgical technique used, length gained and the complications encountered to achieve optimal length. Complications reported included loss of range of motion (ROM) in the digit, pin site infections, transient sensory disturbances, premature consolidation of the callus and post-lengthening fracture through the regenerate bone $[2,4-10]$. One aspect that has not been described in these case reports is the rehabilitation required postoperatively to optimize functional and aesthetic results. Therefore, the purpose of this paper is to provide a detailed case report of the rehabilitation protocol post-distraction osteogenesis of the third metacarpal in a 13-year-old girl with congenital brachymetacarpia. 


\section{Case report}

Written informed consent was attained from both the patient and parents to describe their experience of the rehabilitation process post-distraction osteogenesis for brachymetacarpia. This 13-year-old girl first presented to our institution's plastic and reconstructive surgery clinic at 12 years of age due to episodes of intense pain with subsequent swelling in her right third finger (D3) MPJ. The family described that they first noticed the difference in length of her D3 2 years prior, but there were no functional concerns. Preoperatively, her passive and active ROM in her D3 was MPJ $\left(0^{\circ} / 60^{\circ}\right)$, PIPJ $\left(0^{\circ} / 95^{\circ}\right)$, DIPJ $\left(0^{\circ} / 90^{\circ}\right)$. Pain is atypical in patients with brachymetacarpia as it has not been described in large cohorts with this condition [1]. However, pain in the affected digit appeared to increase over time with greater writing demands at school and participation in competitive baton twirling. As such, her pain was best described as idiopathic musculoskeletal pain that was clearly triggered by activities with high demand and repetitive use of her intrinsic muscles. Her pain was related to overuse of her affected hand, resulting in pain at rest, and with activity. She rated her worse pain intensity in the prior week as 6 out of 10 on the numeric rating scale (NRS). The decision to undergo distraction osteogenesis was made in October 2013. The family's primary goal in pursing treatment was to halt the increasing dysfunction caused primarily by pain.

\section{Surgical intervention}

A Biomet mini external fixator lengthening device was applied to the right D3 with a 4-pin construct (3-mm nonHA-coated steel pins) with a percutaneous technique using a 3-mm incision. The half pins were inserted in a bi-cortical fashion perpendicular to the long axis of the metacarpal, by hand, using a predrilled $1.6 \mathrm{~mm}$ pilot hole with minimal protrusion of the tip of the pin. A proximal reference pin was inserted first, followed by insertion of the most distal pin to ensure that further intermediate pins were aligned with the axis of the metacarpal. Two further half pins were then inserted in the same fashion. A longitudinal incision was made in the periosteum, and a midshaft osteotomy was made of the metacarpal using a drill to perforate the metacarpal diaphysis prior to low-energy osteotomy with an osteotome. Fluoroscopy and direct vision were used to confirm that the osteotomy was complete. After an initial latency period of 7 days, the initial distraction protocol was set at $0.5 \mathrm{~mm} /$ day with the aim of achieving 15-20 mm of length to optimize symmetry with the unaffected side. The distal segment of the fixator was moved at a rate of $0.25 \mathrm{~mm}$ two times a day. Lengthening of the metacarpal occurred for 61 days, followed by a consolidation period of 23 weeks. A minor readjustment of the metacarpal fixator was performed under general anesthesia at day 74 to improve the alignment of the distal fragment that had translated dorsally. No complications ensued after this minor readjustment. Oral cephalexin was used to manage two pin site infections at 26 and 47 days postoperatively.

\section{Rehabilitation}

A functional approach to hand rehabilitation was applied to achieve the goals of full participation in written productivity and baton twirling without pain. The planned therapy program had more frequent follow-up than a traditional block of hand therapy in anticipation of the complications associated with distraction lengthening. The overall rehabilitation timeline for the postoperative first year is shown in Fig. 1. During the 9-week lengthening period, weekly radiographs and clinic appointments with the surgical and rehabilitation teams were necessary to monitor progress. The initial appointment focused on patient and family education regarding the care of the external fixator (ex-fix) hardware. The family was taught how to loosen the screw clamp-locking

\begin{tabular}{|c|c|c|c|}
\hline Timing of Appointments & \multicolumn{2}{|r|}{ Weekly } & Bi-Monthly \\
\hline Massage & Desensitization & \multicolumn{2}{|c|}{ Deep Massage (Scar Management) } \\
\hline ROM Exercises & \multicolumn{3}{|c|}{ A/PROM hook and full fist $\rightarrow$ Isolated PROM MPJ $\rightarrow$ A/PROM composite extension/flexion $\rightarrow$ Isolated EDC AROM* } \\
\hline Hand Exercises & & Strengthening Extrinsic and Intrinsics & Strengthening and Motor Coordination (Dexterity, Speed) \\
\hline Activity Recommendations & Light & Moderate & Return to Regular Activity \\
\hline Splinting & Night & nposite Extension & \\
\hline
\end{tabular}

Timeline (Weeks)

* General progression of ROM exercises, but many exercises overlapped over time

Fig. 1 Rehabilitation timeline 
bolt and use the Allen key to turn the nut $1 / 4$ rotation twice a day to attain $0.5 \mathrm{~mm} /$ day distraction. After this initial week, this task was not a significant burden on the family or teen.

Weekly hand therapy was required up to 28 weeks postoperatively to address the progressive changes in the anatomical structures. Distraction of the metacarpal started at day 7 postoperatively. At the onset of distraction, paresthesia developed in the affected finger originating from both radial and ulnar digital nerves. Palmar massage was introduced to help desensitize the area for comfort and pain management. The sensory thresholds in both radial and ulnar digital nerves in the affected right D3 remained normal until day 33, but then increased to diminished protective sensation (4.31) as measured by Semmes-Weinstein Monofilaments (SWM). Reports of paresthesia resolved at day 68 , which coincided with the end of distraction and the start of the consolidation period. Sensory thresholds returned to normal (2.83) SWM in the affected digit at 3 weeks into the consolidation period.

Both active ROM and passive ROM exercises were essential until 14 weeks to optimize the length of the intrinsic and extrinsic muscles. Isolated MPJ extension and hook fist passive ROM exercises were initiated at the start of the consolidation period ( 8 weeks) and were required to maintain intrinsic muscle length. A thermoplastic night splint was introduced at 9 weeks as an adjunct to active and passive ROM exercises to lengthen the long flexors of the hand. The wrist and digits were positioned in composite extension to attain a maximal stretch on both flexor digitorum superficialis (FDS) and profundus (FDP). The night splint was discharged at 18 weeks.

After the distraction period was completed, two complications arose that were addressed with intensive hand therapy. First, scar tissue around the extensor digitorum communis (EDC) of the middle digit caused adhesions that affected optimal tendon gliding and length. Deep friction massage was introduced 3 weeks into the consolidation period to address these adhesions. However, the progressive scarring resulted in a swan neck deformity of the D3 and contributed to the eventual volar subluxation of the D3 MPJ. Therefore, therapeutic heat (i.e., paraffin wax) and traction with dorsal glide extension passive ROM exercises of the MPJ were also added to the therapy regime to battle the progressive subluxation of the joint. In addition, an anti-swan neck splint was worn during the day. Isolated active EDC exercises were essential to work through the scar tissue that was binding the tendon from gliding as well as focal strengthening of the FDS to address the swan neck deformity. Figure 2 illustrates the changes in the MPJ ROM during the course of hand therapy. At the end of hand therapy, the MPJ passive ROM in extension and flexion exceeded the preoperative ROM, but active ROM of both movements was less than that of the contralateral hand. However, by 21 months post-operative, the MPJ subluxation was resolved and full AROM of the

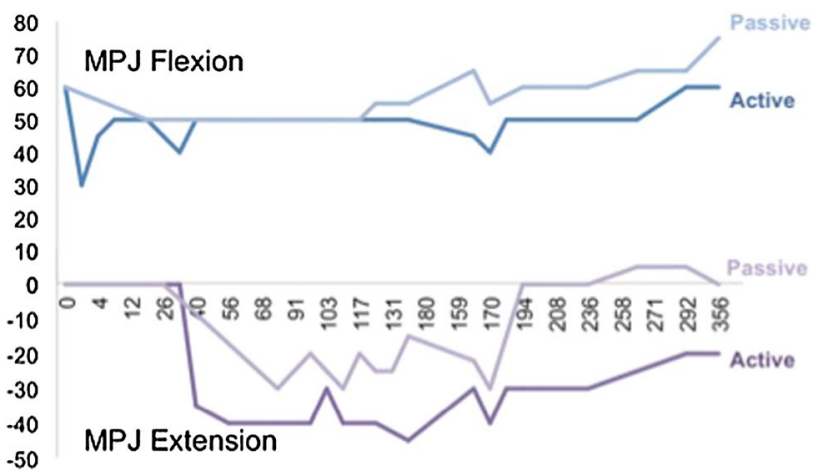

Fig. 2 MPJ ROM

available joint mobility was attained. Her D3 MPJ extension/flexion active and passive ROM was $0^{\circ} / 65^{\circ}$ at her last follow-up visit. She had a $5^{\circ}$ gain in arc of motion from her preoperative examination (pre-operative: $0^{\circ} / 60^{\circ}$ ).

During the course of the rehabilitation, progressive strengthening and motor coordination exercises were prescribed to maintain and improve the strength and speed of dexterity. Qualitative comparison between the in-hand manipulation skills (i.e., translation, shift and rotation with stabilization) of the affected versus the unaffected hand was used to guide treatment. The self-motivation of the teen played an important factor in optimizing the in-hand manipulation function of the hand. As a competitive national level baton twirler, the teen's preoperative advanced dexterity may have influenced her rehabilitation. Therapy sessions typically ended with a baton twirling demonstration or stacking cup competition. Additionally, the teen was attuned to her pain tolerance and took Tylenol prior to therapy sessions.

\section{Outcomes}

The aesthetic outcome gained from distraction osteogenesis of the right middle metacarpal is shown in Fig. 3a and b. Radiographic imaging was taken preoperatively (Fig. 3c) and at 7 months postoperatively (Fig. 3d) immediately after removal of the ex-fix. The overall length in the metacarpal gained was $21 \mathrm{~mm}$. Functionally, the MPJ volar subluxation and swan neck deformity were resolved by the time of the last follow-up visit at 2.5 years from the time of surgery. The teen's pain in her affected digit was also completely resolved; however, she reported using a neoprene glove during long periods of writing (i.e., written exams) for comfort and to prevent pain from fatigue, thus indicating that she was still at risk of pain with overuse. However, the goal of full participation in written productivity and baton twirling without pain was achieved. There were no reports of pain and swelling episodes despite an increasing practice schedule 
Fig. 3 Aesthetic outcome, a preoperative, $\mathbf{b}$ postoperative (1 year), c radiographic Image preoperative, $\mathbf{d}$ radiographic image postoperative ( 7 months)
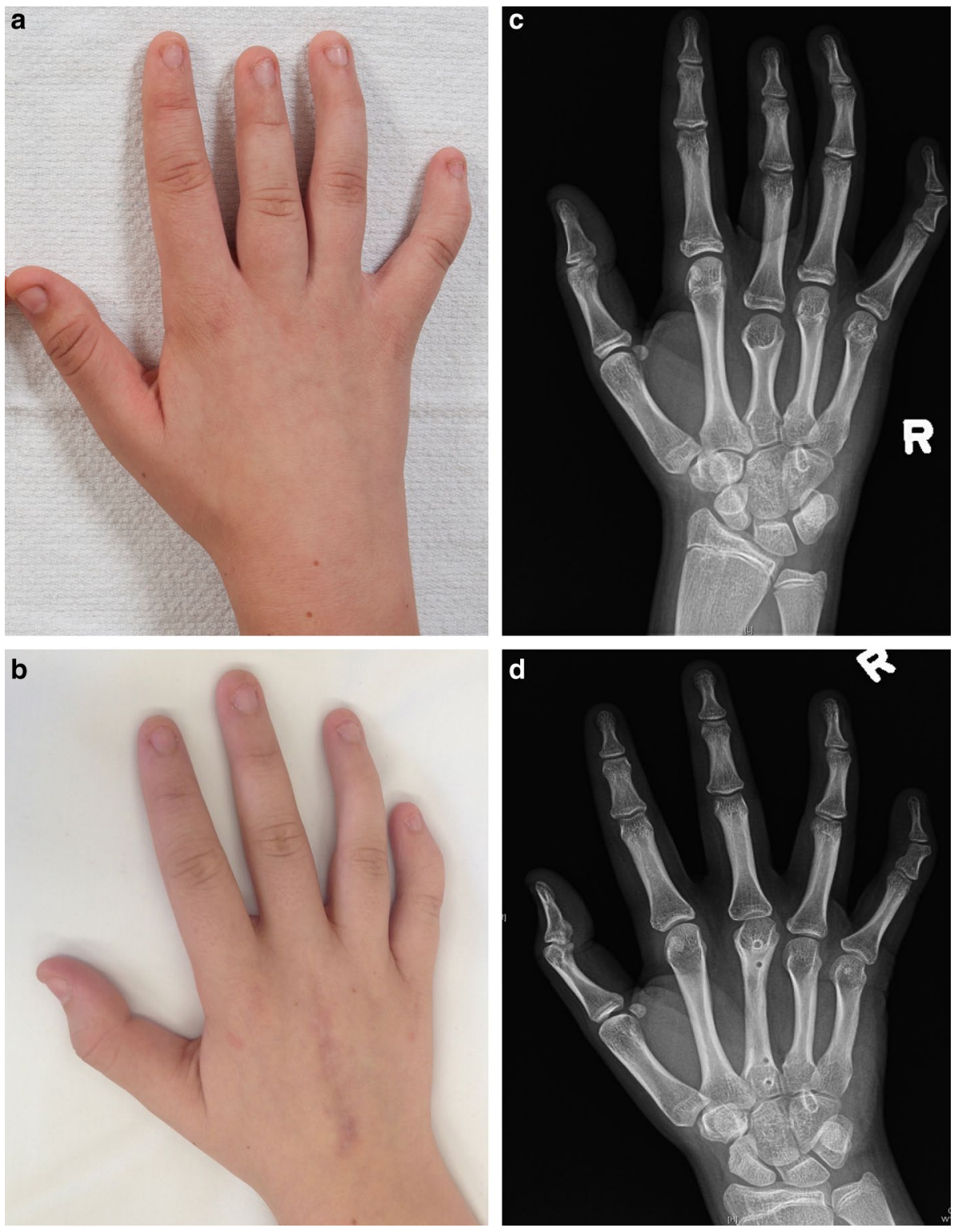

with her baton twirling team. Improved biomechanical alignment of her digits during baton twirling may be an associated benefit of distraction osteogenesis for this teen.

The teen and family reported an overall positive experience with the treatment process. The inconveniences of missing school and the daily aspects of having an ex-fix on one's hand were the reported negative aspects of the treatment. For example, the teen described her frequent frustration with inadvertently getting her ex-fix stuck in her hair when she used the wrong hand to brush (i.e., right hand dominant) her hair. Further, she had a painful experience at the shopping mall when she used a hand drier after washing her hands. The blower directly heated up the ex-fix and caused intense temporary pain. Similarly, cold weather in the winter was uncomfortable as the metal in the ex-fix "froze." Wearing a muff over her hand and hardware was helpful.
Both teen and parents felt that "It probably would have been nice just to have some knowledge of some day to day stuff. There's stuff that we had to figure out, and got around." Better preparation on how to cope and adapt to wearing an exfix at school, home and in the community may improve the child and family's experience.

\section{Discussion}

This case study illustrates that intense hand rehabilitation is required to attain the full benefits of distraction osteogenesis for brachymetacarpia. More specifically, hand therapy was essential to address the complications associated with distraction osteogenesis. The reported complications in the literature including stiffness, loss in ROM, sensory 
disturbances and pin site infections were experienced in this case study $[2,5]$. Frequent follow-up and ongoing adaptation of the therapy program were required to address these complications.

Volpi and Fragomen also reported the need for intense hand therapy to resolve stiffness after distraction osteogenesis due to intrinsic muscle tightness and scarring of the extensor tendons [2]. This case study supports their concerns that maintaining the length of the extrinsic and intrinsic extensors is critical in the rehabilitation of the hand during and after distraction osteogenesis. Therapy and home programming that entails short duration and frequent repetition of therapeutic exercises, deep friction massage and splinting are essential. Earlier efforts to address the progressive scarring and maintain lumbrical muscle and EDC length may have prevented the development of the swan neck deformity and MPJ subluxation. However, these complications occurred quickly. Therapists working with children or adults who undergo distraction osteogenesis for brachymetacarpia should expect to deal with such complications. Prevention of sensory disturbances is unlikely, but educating the teen and family of this expected complication prior to distraction osteogenesis may improve overall care.

The family and teen's commitment to the therapy program as demonstrated by attending regular therapy sessions and following through with home programming was also critical to the success of this procedure. A functional approach to hand rehabilitation is important. In this case study, the teen was motivated to engage in therapy through the use of therapeutic functional activities (i.e., stacking cups, baton twirling) and activity and participation goal setting. They reported an overall positive experience with the treatment process. They reported recognizing that there was pain and hardwork when they opted for the procedure, but the end point to the pain helped them to cope. "I think pain only becomes suffering when there is no point to it... So I don't think she was ever suffering, because she knew that there was going to be an end."

\section{Conclusion}

Distraction osteogenesis for brachymetacarpia has the potential to improve functional and aesthetic outcome. Education regarding the progressive symptoms with distraction and daily challenges of wearing an ex-fix is beneficial to the child and family's experience. As well as, the family and teen's understanding of the intensity of hand therapy and dedication required postoperative be thoroughly assessed before recommending this procedure.

\section{Compliance with ethical standards}

Conflict of interest The authors declare that they have no perceived or actual conflict of interest.

Ethical approval All procedures performed in studies involving human participants were in accordance with the ethical standards of our institutional research ethics board and with the 1964 Helsinki Declaration and its later amendments or comparable ethical standards.

Informed consent Written informed consent was obtained from the child and parent (legal guardian) that participated in this case study. Written informed consent was also obtained from child and parent to use the photographs in this case study.

Open Access This article is distributed under the terms of the Creative Commons Attribution 4.0 International License (http://creativeco mmons.org/licenses/by/4.0/), which permits unrestricted use, distribution, and reproduction in any medium, provided you give appropriate credit to the original author(s) and the source, provide a link to the Creative Commons license, and indicate if changes were made.

\section{References}

1. Miura T, Torii S, Nakamura R (1986) Brachymetacarpia and brachyphalangia. J Hand Surg 11(6):829-836

2. Volpi AD, Fragomen AT (2011) Percutaneous distraction lengthening in brachymetacarpia. Orthopedics 34(8):e424-e427

3. Rayan G, Upton J (2014) Brachymetacarpia. In: Rayan G, Upton $\mathrm{J}$ (eds) Congenital hand anomalies and associated syndromes. Springer, Heidelberg, pp 279-288

4. Smith RJ, Gumley GJ (1985) Metacarpal distraction lengthening. Hand Clin 1(3):417-429

5. Ogino T, Kato H, Ishii S, Usui M (1994) Digital lengthening in congenital hand deformities. J Hand Surg 19(1):120-129

6. Belusa M (1994) Distraction lengthening in brachymetacarpia. Case report. Handchir Mikrochir Plast Chir 26(6):298-301

7. Valenti D, Salazard B, Samson P, Legre R, Magalon G (2006) Bone lengthening by distraction of the metacarpal or phalanx in children. Chir Main 25(6):298-302

8. Saito H, Koizumi M, Takahashi Y, Ohi H (2001) One-stage elongation of the third or fourth brachymetacarpia through the palmar approach. J Hand Surg 26(3):518-524

9. Matev IB (1980) Thumb reconstruction through metacarpal bone lengthening. J Hand Surg 5(5):482-487

10. Bozan ME, Altinel L, Kuru I, Maralcan G, Acar M, Durmaz H (2006) Factors that affect the healing index of metacarpal lengthening: a retrospective study. J Orthop Surg 14(2):167-171 